\title{
Strain Estimates for Small-Ring Cyclic Allenes and Butatrienes
}

\author{
Kimberly J. Daoust, Susanna M. Hernandez, Kaleen M. Konrad, \\ Iain D. Mackie, James Winstanley Jr. and Richard P. Johnson* \\ Department of Chemistry \\ University of New Hampshire \\ Durham, New Hampshire 03824 \\ rpj@cisunix.unh.edu \\ Supplementary Material
}

\section{Contents}

B3LYP/6-311+G(d,p) optimized structures and energies 


\title{
B3LYP/6-311+G(d,p) optimized structures and energies Energies in hartrees; geometries in Angstroms.
}

\author{
1,2-cyclobutadiene ${ }^{1} \mathrm{~A}_{2}$ electronic state $\mathrm{C} 2 \mathrm{v}$ \\ C,0,0.815426175,0.,-0.7115963096 \\ C,0,0.8172076645,0.,0.6749946686 \\ H,0,1.5503897982,0.,1.4652109144 \\ C,0,-0.558161357,0.,-0.9010560855 \\ H,0,-1.2418578254,0.,-1.7344568867 \\ C,0,-0.7272640496,0.,0.6346600461 \\ H,0,-1.1958912853,-0.9001968175,1.0436160274 \\ H,0,-1.1958912853,0.9001968175,1.0436160274 \\ UB3LYP Energy $=-154.674843174 \quad \mathrm{~S}^{* * 2}=1.0439$ \\ Zero-point correction= \\ 0.059436 \\ Thermal correction to Energy= 0.063382 \\ Thermal correction to Enthalpy= $\quad 0.064326$ \\ Thermal correction to Gibbs Free Energy $=\quad 0.034681$ \\ Sum of electronic and zero-point Energies= $\quad-154.615407$ \\ Sum of electronic and thermal Energies $=\quad-154.611462$ \\ Sum of electronic and thermal Enthalpies $=\quad-154.610517$ \\ Sum of electronic and thermal Free Energies $=\quad-154.640162$
}

\section{1,2-cyclopentadiene}

UB3LYP Energy =-194.0504399 S2=0.805141

Zero-point correction $=\quad 0.089328$

Thermal correction to Energy= $\quad 0.094026$

Thermal correction to Enthalpy= $\quad 0.094970$

Thermal correction to Gibbs Free Energy= $\quad 0.062815$

Sum of electronic and zero-point Energies $=\quad-193.961112$

Sum of electronic and thermal Energies $=\quad-193.956414$ 
Sum of electronic and thermal Enthalpies $=\quad-193.955470$

Sum of electronic and thermal Free Energies $=\quad-193.987625$

C,0.5624567551,0.0454905604,-1.1484152099

C,0.5624567553,-0.0454905603,1.1484152097

C,1.3054246006,0.0000000002,-0.0000000001

H,0.9030394249,-0.1438729014,-2.1580062354

H,0.9030394254,0.1438729016,2.1580062352

C,-0.8988009555,0.2017858005,-0.757357496

H,-1.5386320531,-0.4360899822,-1.3745075499

H,-1.2626159727,1.2306481323,-0.8820003833

C,-0.8988009553,-0.2017858007,0.7573574962

H,-1.2626159722,-1.2306481326,0.8820003835

H,-1.5386320529,0.4360899819,1.3745075502

\section{1,2-cyclohexadiene ( $\mathrm{C}_{1}$ symmetry)}

B3LYP Energy =-233.4145041 S2=0.0

Zero-point correction=

0.120409

Thermal correction to Energy= $\quad 0.125926$

Thermal correction to Enthalpy= $\quad 0.126870$

Thermal correction to Gibbs Free Energy= 0.091694

Sum of electronic and zero-point Energies $=\quad-233.294095$

Sum of electronic and thermal Energies $=\quad-233.288578$

Sum of electronic and thermal Enthalpies= $\quad-233.287634$

Sum of electronic and thermal Free Energies $=\quad-233.322810$

Charge $=0$ Multiplicity $=1$

C,0,0.8748377952,-0.0746443808,-1.208286036

C,0,0.8714345034,-0.0874895169,1.2125495588

C,0,1.3958491863,-0.0800327583,0.0022601571

H,0,1.2671671241,-0.6106490266,-2.0654872975

H,0,1.2373532069,0.4714841387,2.0669258248

C,0,-0.543271076,0.5048694415,-1.2515291931 
H,0,-1.0716213891,-0.0214257963,-2.053977219

H,0,-0.5575527393,1.5647773729,-1.522002002

C,0,-0.5576048598,-0.6174609201,1.1530350873

H,0,-0.5833229406,-1.6596009708,0.8269371595

H,0,-1.0636867174,-0.5582956868,2.1204408722

C,0,-1.2906537428,0.2981520218,0.1130543442

H,0,-1.448466273,1.2701883107,0.5879162084

H,0,-2.2834211101,-0.116841665,-0.0872570558

\section{1,2-cycloheptadiene}

B3LYP Energy =-272.7582127

Zero-point correction=

0.149731

Thermal correction to Energy=

0.156154

Thermal correction to Enthalpy=

0.157098

Thermal correction to Gibbs Free Energy=

0.119970

Sum of electronic and zero-point Energies=

$-272.608481$

Sum of electronic and thermal Energies=

$-272.602059$

Sum of electronic and thermal Enthalpies $=\quad-272.601115$

Sum of electronic and thermal Free Energies $=\quad-272.638242$

Charge $=0$ Multiplicity $=1$

C,0,1.1559931465,-0.0185491875,-1.2622195514

C,0,1.1567931444,0.0172372315,1.2614884674

C,0,1.5034514961,-0.0008480257,-0.0004726203

$\mathrm{H}, 0,1.6427659436,-0.6314843703,-2.014778177$

$\mathrm{H}, 0,1.6446457721,0.6297039033,2.0137318873$

C,0,-0.1773984029,0.6619975712,-1.5608974258

H,0,-0.3999117248,0.6113524841,-2.6310330172

H,0,-0.1408134335,1.7225718229,-1.2919428787

C,0,-0.1771672302,-0.6618508283,1.5609796128

H,0,-0.3989221994,-0.6111309458,2.631268572

C,0,-1.3158684979,-0.0455583513,-0.7761267478 
H,0,-1.3258163334,-1.103811119,-1.066139146

H,0,-2.2697154943,0.3727074495,-1.1192285328

C,0,-1.3153135061,0.0471007218,0.7770041073

H,0,-1.3237939433,1.1053599709,1.0670424623

H,0,-2.2694418007,-0.3700245424,1.1207120515

H,0,-0.1419376854,-1.7224194428,1.2918317251

\section{1,2-cyclooctadiene}

B3LYP Energy =-312.0967199

Zero-point correction=

0.178623

Thermal correction to Energy=

0.186190

Thermal correction to Enthalpy=

0.187135

Thermal correction to Gibbs Free Energy=

0.147276

Sum of electronic and zero-point Energies=

$-311.918097$

Sum of electronic and thermal Energies=

$-311.910530$

Sum of electronic and thermal Enthalpies= $-311.909585$

Sum of electronic and thermal Free Energies= $-311.949444$

Charge $=0$ Multiplicity $=1$

C,0,1.3579754304,-0.0522927845,-1.3674948475

C,0,1.3022032714,-0.0496464889,1.2091561286

C,0,1.5397991085,-0.0526751471,-0.0745947223

H,0,2.0199542244,-0.5869789192,-2.0440204304

H,0,1.771998125,0.6764585197,1.8684392353

C,0,0.1287286543,0.630522647,-1.9457418941

H,0,-0.3023436364,-0.0506515032,-2.6914398547

H,0,0.4120145764,1.5351646144,-2.498848527

C,0,0.1894241888,-0.9274904826,1.7558116437

H,0,0.270556686,-1.9364564075,1.3401659854

H,0,0.2747074304,-1.0215893939,2.842702459

C,0,-1.1984224529,-0.3426086183,1.4008623167

H,0,-1.3063556151,0.6420855798,1.8723783067 
H,0,-1.9550951114,-0.9852023918,1.8669321104

C,0,-0.9437433783,0.9833584789,-0.8981108622

H,0,-1.7547174837,1.4926970269,-1.4285822293

H,0,-0.5358009996,1.7201909223,-0.1992522695

C,0,-1.5401739671,-0.2192147727,-0.1052790667

H,0,-1.2788190131,-1.1574656875,-0.6088773241

H,0,-2.6308443143,-0.1479693509,-0.1672496403

\section{1,2-cyclononadiene}

B3LYP Energy =-351.4229811

Zero-point correction= 0.207384

Thermal correction to Energy= 0.216186

Thermal correction to Enthalpy= 0.217130

Thermal correction to Gibbs Free Energy= 0.174311

Sum of electronic and zero-point Energies= $-351.215597$

Sum of electronic and thermal Energies= $-351.206795$

Sum of electronic and thermal Enthalpies= $-351.205851$

Sum of electronic and thermal Free Energies= $-351.248670$

Charge $=0$ Multiplicity $=1$

H,0,-0.3400606831,-1.0101913278,-2.8223995025

C,0,-0.3465778278,-0.9924130484,-1.7340579912

C,0,0.7724096494,-0.9942426544,-1.0635546292

C,0,1.7707405539,-0.8585317941,-0.2323668081

H,0,2.2686945854,-1.7455217397,0.1551674147

C,0,2.1839562465,0.490605901,0.3329398411

H,0,2.390620861,1.1976754525,-0.4771339784

H,0,3.1176857662,0.3731971346,0.8906139593

C,0,1.1027788073,1.0889271015,1.263394718

H,0,0.8604988133,0.3553279194,2.0408697022

H,0,1.5482225632,1.9423571207,1.7859354621

C,0,-0.1962061075,1.5533731342,0.5468553784 
H,0,-0.047483065,1.5351986982,-0.5355099568

H,0,-0.3672372864,2.605166074,0.8005632402

C,0,-1.4955184751,0.801084526,0.9035814215

H,0,-2.3348067849,1.3706901787,0.4840378775

H,0,-1.6260659492,0.843212371,1.9925277801

C,0,-1.6630427661,-0.6705340156,0.4700011868

H,0,-2.6116739177,-1.0184841814,0.8934190823

H,0,-0.8862054835,-1.2927207389,0.9256412798

C,0,-1.7019644971,-0.93674999647,-1.046169024

H,0,-2.2275682152,-1.8815137559,-1.2353276654

H,0,-2.3040747047,-0.1635083186,-1.542149255

\section{1,2,4-cyclohexatriene}

C,0,-0.7522138213,-0.0085091531,-1.1494744033

C,0,-0.7539610664,-0.0046113674,1.1972067353

C,0,1.3966959613,-0.0073480996,-0.1397422102

C,0,0.5899530622,0.0620184506,1.1599718685

C,0,0.5051547805,0.3791054107,-1.3083189018

C,0,-1.4186226002,-0.400910152,-0.0683378907

H,0,2.2123384177,0.7227770293,-0.1000209314

H,0,-2.1943494932,-1.1601608222,-0.0534510443

$\mathrm{H}, 0,-1.2981173767,0.0442231481,2.134330999$

H,0,1.8904577878,-0.9890689337,-0.1997262734

H,0,1.1578327007,0.125715434,2.0833872527

H,0,0.8298000672,1.138043609,-2.0123511896

B3LYP Energy $=-232.180376401 \quad S^{* * 2}=0.0000$

Zero-point correction=

0.096459

Thermal correction to Energy=

0.101477

Thermal correction to Enthalpy=

0.102421 
Thermal correction to Gibbs Free Energy=

Sum of electronic and zero-point Energies=

Sum of electronic and thermal Energies=

Sum of electronic and thermal Enthalpies=

Sum of electronic and thermal Free Energies=
0.068556

$-232.083917$

$-232.078900$

$-232.077956$

$-232.111820$

\section{bicyclo[3.2.1]octa-2,3-diene}

H,0,-0.6475183646,-0.98670498,-1.8333287372

C,0,-0.6477027767,-0.9884477111,-0.741254275

C,0,0.7993762618,-0.9888326933,-0.1273298079

C,0,0.5163281005,-0.5865413826,1.3560576729

C,0,-0.9312852577,-0.0035314484,1.3887881919

C,0,-1.2504814742,0.2802437821,-0.1124210977

H,0,-1.2081948728,-1.8654484256,-0.4006432678

H,0,1.3033347587,-1.953590601,-0.204359364

H,0,0.6008683274,-1.4600575241,2.0058165754

H,0,1.2475166913,0.146616399,1.6994079353

H,0,-1.0081595313,0.8855501155,2.01798066

H,0,-1.6448281493,-0.7394050693,1.7738867635

H,0,-2.3132037838,0.4160859287,-0.3228126518

C,0,-0.3740245856,1.5291050648,-0.3791454125

H,0,-0.6083714902,2.4355739659,0.1713789069

C,0,1.4489045296,0.0780288769,-1.0070068743

H,0,2.0476453415,-0.2128534045,-1.865007123

C,0,0.8107037146,1.2356811108,-0.8847416803

B3LYP Energy $=-310.831388038 \mathrm{~S} * * 2=0.0000$

Zero-point correction=

Thermal correction to Energy=

Thermal correction to Enthalpy=

\subsection{4}

0.162613

0.163557 
Thermal correction to Gibbs Free Energy=

0.127027

Sum of electronic and zero-point Energies $=\quad-310.674834$

Sum of electronic and thermal Energies $=\quad-310.668775$

Sum of electronic and thermal Enthalpies $=\quad-310.667831$

Sum of electronic and thermal Free Energies $=\quad-310.704361$

\section{1,3-cyclobutadiene}

B3LYP ENERGY = -154.7211603

Zero-point correction=

0.060893

Thermal correction to Energy=

0.064675

Thermal correction to Enthalpy=

0.065619

Thermal correction to Gibbs Free Energy=

0.036902

Sum of electronic and zero-point Energies=

$-154.660268$

Sum of electronic and thermal Energies=

$-154.656485$

Sum of electronic and thermal Enthalpies=

$-154.655541$

Sum of electronic and thermal Free Energies=

$-154.684259$

H,0,-0.0964289951,0.,-2.1095655825

C,0,-0.0929547499,0.,-1.0284487003

C,0,1.0295731687,0.,0.0795387013

C,0,-1.0295731687,0.,-0.0795387013

C,0,0.0929547499,0.,1.0284487003

H,0,0.0964289951,0.,2.1095655825

H,0,-2.1106434628,0.,-0.0689178542

H,0,2.1106434628,0.,0.0689178542

\section{1,3-cyclopentadiene}

B3LYP ENERGY = -194.156236

Zero-point correction=

0.092119

Thermal correction to Energy=

0.096277

Thermal correction to Enthalpy=

0.097221

Thermal correction to Gibbs Free Energy=

0.065520 
Sum of electronic and zero-point Energies= $-194.064117$

Sum of electronic and thermal Energies= $-194.059959$

Sum of electronic and thermal Enthalpies= $-194.059015$

Sum of electronic and thermal Free Energies= $-194.090716$

C,0,0.0452686547,0.,1.2316591629

H,0,0.0459744087,0.,2.3141815201

C,0,1.1318043142,0.,0.4350533607

H,0,2.1667339334,0.,0.7486001438

C,0,-1.1653973418,0.,0.4011017087

H,0,-2.1756138677,0.,0.7900974964

C,0,-0.81328567,0.,-0.8993435667

H,0,-1.4783390936,0.,-1.7520421334

C,0,0.6878012988,0.,-1.0025768121

H,0,1.0620485417,-0.8770683483,-1.5481000736

H,0,1.0620485417,0.8770683483,-1.5481000736

\section{1,3-cyclohexadiene}

B3LYP ENERGY = -233.4838769

Zero-point correction= 0.121814

Thermal correction to Energy= 0.126997

Thermal correction to Enthalpy= 0.127941

Thermal correction to Gibbs Free Energy=

0.094232

Sum of electronic and zero-point Energies= $-233.362063$

Sum of electronic and thermal Energies= $-233.356880$

Sum of electronic and thermal Enthalpies= $-233.355935$

Sum of electronic and thermal Free Energies= $-233.389645$

C,0,-0.6561059026,0.0755923148,-1.2669076551

C,0,-0.6555037481,0.0774223619,1.2561399728

C,0,1.4529507918,0.0711653674,-0.0402299303

C,0,0.8460866197,-0.044133186,1.1504059518

C,0,0.6695098684,0.2802953722,-1.2613247007 
C,0,-1.3580547992,-0.4108498734,-0.021367286

H,0,-0.9051214969,1.1354650022,1.4335693626

H,0,2.5347608473,0.0327757569,-0.1156131944

H,0,1.427101793,-0.1611972721,2.0596492302

H,0,1.186137969,0.5833722238,-2.1661502212

H,0,-1.3695328341,-1.5121276695,-0.0386851446

H,0,-1.2313411728,0.1936135018,-2.1796953383

H,0,-1.0297870537,-0.4721241675,2.1243376879

H,0,-2.4055150321,-0.096731517,-0.017710497

\section{1,3-cycloheptadiene}

B3LYP ENERGY $=-272.8059512$

Zero-point correction=

0.150823

Thermal correction to Energy=

0.157265

Thermal correction to Enthalpy=

0.158209

Thermal correction to Gibbs Free Energy=

0.120071

Sum of electronic and zero-point Energies= $-272.655128$

Sum of electronic and thermal Energies= $-272.648686$

Sum of electronic and thermal Enthalpies= $-272.647742$

Sum of electronic and thermal Free Energies= $-272.685880$

C,0,0.0816015618,0.066258279,1.6659026821

H,0,0.0831441413,0.0657264616,2.7534143309

C,0,1.282356311,0.0662187407,1.0634942866

H,0,2.1470639865,0.0571046464,1.7224432915

C,0,-1.2812358607,0.0649538129,1.0314569779

H,0,-1.6564823192,1.0973271275,0.9826214041

C, $0,-0.673684383,0.1498580519,-1.4944496452$

H,0,-0.9696285331,-0.3143989147,-2.4428065777

C, $0,0.8284303499,0.1706262746,-1.4390519624$

C,0,1.6243365466,0.1140098708,-0.3582949346

H,0,1.3238278738,0.2391098079,-2.4047514745 
H,0,2.6940385903,0.133543375,-0.5516138468

H,0,-1.9685581039,-0.4539975466,1.7102617826

H,0,-1.045878114,1.1826579798,-1.5559769907

C,0,-1.3873118712,-0.587022067,-0.3534192559

H,0,-1.0055956428,-1.6118781503,-0.2960546521

H,0,-2.4488878051,-0.6646125637,-0.6113661583

\section{1,3-cyclooctadiene}

B3LYP ENERGY = -312.1253129

Zero-point correction=

0.179605

Thermal correction to Energy=

0.186976

Thermal correction to Enthalpy=

0.187920

Thermal correction to Gibbs Free Energy=

0.148432

Sum of electronic and zero-point Energies=

$-311.945708$

Sum of electronic and thermal Energies=

$-311.938337$

Sum of electronic and thermal Enthalpies= $-311.937393$

Sum of electronic and thermal Free Energies= $-311.976881$

C,0,0.2498460745,0.8259956995,1.5248485219

H,0,0.2624034085,0.8379681039,2.612825906

C,0,1.4328234645,0.8512060933,0.8925714581

H,0,2.3357813647,0.9431442495,1.4939642552

C,0,1.615063228,0.8072071307,-0.560547627

H,0,2.2695208087,1.5626881288,-0.9922815148

C,0,1.0734627048,-0.1059639525,-1.3806892154

H,0,1.2713005162,-0.0034685553,-2.4457483309

C,0,0.2707660349,-1.3161362516,-0.9743683964

H,0,0.6545695037,-1.7087805346,-0.0264969515

H,0,0.4359403651,-2.0926238071,-1.7277353691

C,0,-1.2563700587,-1.0988499427,-0.8328559892

H,0,-1.7527979947,-2.0658504398,-0.9685734444

H,0,-1.6074099594,-0.4571543581,-1.6498414803 
C,0,-1.7177992806,-0.5194453614,0.5088552983

H,0,-1.5057386073,-1.2367609191,1.3106997307

H,0,-2.807654043,-0.4155620687,0.4717608945

C,0,-1.1140995044,0.8572600514,0.882504043

H,0,-1.7982557425,1.3513560159,1.5793355864

H,0,-1.0798155976,1.4774033838,-0.0198178427

\section{1,3-cyclononadiene}

B3LYP ENERGY = -351.4413051

Zero-point correction= 0.207859

Thermal correction to Energy= 0.216550

Thermal correction to Enthalpy= 0.217494

Thermal correction to Gibbs Free Energy= 0.174835

Sum of electronic and zero-point Energies= $-351.233446$

Sum of electronic and thermal Energies= $-351.224755$

Sum of electronic and thermal Enthalpies= $-351.223811$

Sum of electronic and thermal Free Energies= $-351.266470$

C,0,0.3233710557,1.1144584141,1.4124825535

H,0,0.3261492859,1.1237876613,2.5014353741

C,0,1.502453291,1.1049490274,0.7769875931

H,0,2.4151961997,1.1554568956,1.3693961846

C,0,1.6574544234,1.1056032084,-0.6916381067

H,0,2.1095352213,1.9965699689,-1.1268207531

C,0,1.2918439657,0.1172968345,-1.5173168954

C,0,-0.7451259892,-1.4678051945,-0.9834404766

H,0,-1.2870715168,-0.7711417451,-1.6328650486

C,0,-1.2914390545,-1.3977346769,0.4632091499

H,0,-0.4887692214,-1.6786177169,1.1541026702

H,0,-2.0643460608,-2.1650392663,0.5819024826

C,0,-1.9079765435,-0.0654563138,0.9177304536

H,0,-2.1952356842,-0.1658301564,1.9717381674 
H,0,-2.8402332954,0.1067307296,0.3660867017

C,0,-1.0232256622,1.183036575,0.7452693833

H,0,-1.5693189932,2.0400143416,1.1606949105

H,0,-0.887668932,1.3876187249,-0.3189696138

H,0,1.4238937831,0.283984945,-2.58477433939

H,0,-0.9661064357,-2.4663637491,-1.372048458

C,0,0.7925521315,-1.2524198015,-1.1242250834

H,0,1.263593955,-1.5476755695,-0.1817886785

H,0,1.1609359876,-1.9510634995,-1.8824409737

\section{Cyclobutane}

B3LYP ENERGY = -157.257205

Zero-point correction=

0.110251

Thermal correction to Energy=

0.114401

Thermal correction to Enthalpy=

0.115345

Thermal correction to Gibbs Free Energy=

0.084749

Sum of electronic and zero-point Energies= $-157.146954$

Sum of electronic and thermal Energies= $-157.142804$

Sum of electronic and thermal Enthalpies= $-157.141860$

Sum of electronic and thermal Free Energies= $-157.172456$

H,0,-0.4540830839,0.5832506957,-1.8914548646

C,0,-0.4571807996,0.5869965628,-0.7994932509

C,0,0.9164424159,0.5896129057,-0.0725163855

C,0,-0.7958460965,-0.7443779984,-0.0727948674

C, $0,0.3365844801,-0.4322314701,0.9448045039$

H,0,0.9793568603,-1.2573512408,1.2585318126

H,0,-0.0503720245,0.0642792584,1.8384607493

H,0,-1.0953712445,1.4060895223,-0.4579095793

H,0,-1.8087283832,-0.867460596,0.3162089119

H,0,-0.5542419946,-1.6132866308,-0.6904557545

H,0,1.2834546068,1.541561141,0.3167141401 
H,0,1.6999852635,0.1429178502,-0.6900954155

\section{Cyclopentane}

B3LYP ENERGY = -196.611661

Zero-point correction $=\quad 0.140042$

Thermal correction to Energy $=\quad 0.145224$

Thermal correction to Enthalpy= $\quad 0.146168$

Thermal correction to Gibbs Free Energy= $\quad 0.111175$

Sum of electronic and zero-point Energies $=\quad-196.471619$

Sum of electronic and thermal Energies $=\quad-196.466437$

Sum of electronic and thermal Enthalpies= $\quad-196.465493$

Sum of electronic and thermal Free Energies $=\quad-196.500486$

H,0,-0.3693494939,-0.6025577644,-2.1625370199

C,0,-0.3727563906,-0.607318245,-1.0693396594

C,0,1.0357302678,-0.6120445247,-0.4566335223

C,0,0.7978291683,-0.1905201507,1.0079231462

C,0,-0.4419948549,0.7511376751,0.9748191081

C,0,-1.0168402233,0.6535189562,-0.4652005386

H,0,-0.9192092086,-1.5019972867,-0.7477733062

H,0,1.6594314792,0.1306913368,-0.9676972837

H,0,1.5428917648,-1.5764865812,-0.5433027953

H,0,0.5807622879,-1.0754337371,1.6141131126

H,0,1.6744111047,0.2881495322,1.4509916408

H,0,-0.1723830412,1.7810405765,1.2210628404

H,0,-1.1827347464,0.4376830977,1.7148871416

$\mathrm{H}, 0,-0.7166502797,1.5297712431,-1.0493315814$

H,0,-2.1089776705,0.6204973178,-0.4798239538

\section{cyclohexane}

B3LYP ENERGY $=-235.9448331$

Zero-point correction $=\quad 0.169497$ 
$\begin{array}{lc}\text { Thermal correction to Energy= } & 0.175229 \\ \text { Thermal correction to Enthalpy= } & 0.176173 \\ \text { Thermal correction to Gibbs Free Energy= } & 0.140709 \\ \text { Sum of electronic and zero-point Energies= } & -235.775336 \\ \text { Sum of electronic and thermal Energies= } & -235.769604 \\ \text { Sum of electronic and thermal Enthalpies= } & -235.768660 \\ \text { Sum of electronic and thermal Free Energies= } & -235.804124 \\ \text { H,0,-0.5430981482,-0.7417675037,-2.3241510449 } & \text { C,0,-0.4886195543,--0.6674162315,-1.23299477 } \\ \text { C,0,1.085355176,-0.6675691204,0.7611373195 } \\ \text { C,0,-0.9639434419,0.8339472201,0.7613472429 } \\ \text { C,0,0.4886248175,0.6674130867,1.2329956231 } \\ \text { C,0,-1.0853601681,0.6675735601,-0.7611416769 } \\ \text { C,0,0.9639471446,-0.833949516,-0.7613421811 } \\ \text { H,0,0.556226316,-1.4925211545,1.2561770473 } \\ \text { H,0,-1.5907551114,0.0804931454,1.2564349014 } \\ \text { H,0,1.0929546614,1.492725378,0.8339317097 } \\ \text { H,0,-0.556231279,1.4925304358,-1.256178149 } \\ \text { H,0,1.5907488314,-0.0804928522,-1.2564324082 } \\ \text { H,0,-1.0929746292,-1.4927209324,-0.8339484188 } \\ \text { H,0,2.1339105971,-0.7426052401,1.0677755452 } \\ \text { H,0,-1.3519990392,1.811009562,1.0678093582 } \\ \text { H,0,0.5431088774,0.7417800348,2.3241494168 } \\ \begin{array}{l}\text { H,0,-2.1339067698,0.7425866032,-1.0677655403 } \\ \text { H,0,1.3519918509,-1.8110114706,-1.0678117622 }\end{array}\end{array}$

\section{cycloheptane}

B3LYP ENERGY = -275.2585949

Zero-point correction=

Thermal correction to Energy=

Thermal correction to Enthalpy=
0.198089

0.205171

0.206115 
Thermal correction to Gibbs Free Energy=

Sum of electronic and zero-point Energies=

Sum of electronic and thermal Energies=

Sum of electronic and thermal Enthalpies=

Sum of electronic and thermal Free Energies=

H,0,1.6853155745,-0.0650370824,-1.479756274

C,0,1.6795824773,-0.0656199752,-0.3816121896

H,0,2.7324580447,-0.064926755,-0.0761461009

C,0,1.0212996209,1.2420885113,0.090905849

H,0,1.6858080095,2.0687109379,-0.1834270803

H,0,0.9563330887,1.2607293919,1.1864795959

C,0,-0.3614751112,1.5015021657,-0.534462734

H,0,-0.552812086,2.579709082,-0.557546101

H,0,-0.3187897401,1.1899445518,-1.585040362

C,0,-1.5655088131,0.8260717587,0.153303075

H,0,-2.3953617066,0.8113565617,-0.5626915958

H,0,-1.903295004,1.4569148651,0.9836392688

C,0,-1.3487947452,-0.5990443728,0.7019415918

H,0,-0.9101801457,-0.5414542293,1.7053877283

H,0,-2.3344751143,-1.0561747553,0.8402848518

C,0,-0.4669857646,-1.5345832059,-0.1451872544

H,0,-0.684757973,-1.4040313852,-1.2130736974

H,0,-0.7352458276,-2.5702939164,0.0903428995

C,0,1.040206223,-1.3695304476,0.1152757963

H,0,1.2098761419,-1.462668219,1.1962434704

H,0,1.5751834154,-2.2080856529,-0.3456814072
0.167961

$-275.060506$

$-275.053424$

$-275.052480$

$-275.090634$

\section{cyclooctane}

B3LYP ENERGY = -314.5761069

Zero-point correction= 0.227259

Thermal correction to Energy=

0.235308 
Thermal correction to Enthalpy=

Thermal correction to Gibbs Free Energy=

Sum of electronic and zero-point Energies=

Sum of electronic and thermal Energies=

Sum of electronic and thermal Enthalpies=

Sum of electronic and thermal Free Energies=

H,0,1.418788076,-0.0174261497,-2.3808377647

C,0,1.4221241967,-0.0148518875,-1.2837663181

H,0,2.4790952606,-0.0172763571,-0.9892200306

C,0,0.7719254985,-1.3292263312,-0.8033210018

H,0,1.1876027309,-2.1353788317,-1.4170114743

H,0,1.0879203544,-1.5548393133,0.2193423511

C,0,-0.771846815,-1.3857636902,-0.8804587911

H,0,-1.0662043713,-2.3855638047,-1.217109468

H,0,-1.1343876079,-0.6997706675,-1.6557278271

C,0,-1.5222954687,-1.1269898514,0.4398281407

H,0,-1.1858978416,-1.8721853563,1.1733113771

H,0,-2.5842229349,-1.3389586751,0.2669059871

C,0,-1.4227289245,0.2640911601,1.0942552081

H,0,-2.109310611,0.2585000954,1.9475749874

H,0,-1.8154201269,1.0265093891,0.410459628

C,0,-0.0192390481,0.6762161994,1.5969964802

H,0,0.5631194995,-0.22457501,1.8115378961

H,0,-0.123230751,1.187319621,2.5599317997

C,0,0.7781474647,1.617499224,0.6699974273

H,0,0.3741326365,2.6305646596,0.7885468148

H,0,1.8147344467,1.66674429,1.0263870674

C,0,0.7730219996,1.3015559585,-0.8326432191

H,0,-0.2559639628,1.3419832837,-1.2055821727

H,0,1.2945917831,2.1191681373,-1.3438367279
0.195403

$-314.348848$

$-314.340799$

$-314.339855$

$-314.380704$

(1)


cyclononane (Note: see footnote 32 for explanation)

B3LYP ENERGY = -353.8947127

C,0,-0.1484253003,-0.0398482456,1.8247869156

H,0,-0.1396495753,-0.035556292,2.9217087323

H,0,0.9045573145,-0.0333709303,1.5336279508

C,0,-0.7974014557,-1.3644149986,1.3914636092

H,0,-1.83886635835,-1.375107051,1.7386611308

H,0,-0.2944952329,-2.1672827095,1.9455571586

C,0,-0.7875420132,-1.7578063054,-0.1000175731

H,0,-1.4929629091,-1.1441313996,-0.6663568189

H,0,-1.1971659492,-2.7731011924,-0.14776152

C,0,0.5819724306,-1.7630371607,-0.8201992871

H,0,0.6004672465,-2.6389063332,-1.4776560713

H,0,1.3846052319,-1.933037999,-0.0909375988

C,0,0.9521961177,-0.5506432932,-1.7062421746

H,0,0.1024928135,-0.3095489228,-2.3580345687

H,0,1.7433380729,-0.8954722064,-2.3807282897

C,0,1.4649244134,0.7361445651,-1.0166470943

H,0,2.2747570313,1.1470821895,-1.6300075269

H,0,1.9381378169,0.474747262,-0.0665168851

C,0,0.4523690836,1.8837905223,-0.8244704981

H,0,0.216104245,2.2823299122,-1.819399474

H, 0,0.9604510781,2.6996768385,-0.2939208089

C,0,-0.8849704853,1.5984610359,-0.1219476709

H,0,-1.4299903647,0.8250695697,-0.6681827994

H,0,-1.4962404348,2.5034140443,-0.2256389195

C,0,-0.8365407912,1.262372802,1.3789351672

$\mathrm{H}, 0,-0.346617317,2.0967932896,1.8978300908$

H,0,-1.8684174814,1.2462883995,1.7537878552

Zero-point correction $=\quad 0.255747$ 
Thermal correction to Energy $=\quad 0.265028$

Thermal correction to Enthalpy= $\quad 0.265972$

Thermal correction to Gibbs Free Energy $=\quad 0.222204$

Sum of electronic and zero-point Energies $=\quad-353.638965$

Sum of electronic and thermal Energies $=\quad-353.629685$

Sum of electronic and thermal Enthalpies $=\quad-353.628741$

Sum of electronic and thermal Free Energies $=\quad-353.672508$

\section{1,3-dimethylallene}

B3LYP ENERGY = -195.3514366

Zero-point correction $=\quad 0.111905$

Thermal correction to Energy $=\quad 0.118438$

Thermal correction to Enthalpy= $\quad 0.119382$

Thermal correction to Gibbs Free Energy= $\quad 0.082360$

Sum of electronic and zero-point Energies $=\quad-195.239531$

Sum of electronic and thermal Energies $=\quad-195.232999$

Sum of electronic and thermal Enthalpies= $\quad-195.232054$

Sum of electronic and thermal Free Energies $=\quad-195.269077$

Charge $=0$ Multiplicity $=1$

C,0,0.2920473876,-0.2932157422,-1.3057806234

C,0,0.2914916077,-0.2923795396,0.0007285595

H,0,1.2526337174,-0.294737515,-1.820786241

C,0,0.2899460905,-0.2897505615,1.3072329716

H,0,0.2895593875,-1.2490435331,1.8246429115

C,0,-0.9408170094,-0.2948218757,-2.1765641672

H,0,-1.8511783423,-0.2975862266,-1.575314017

H,0,-0.9545749923,-1.1753289834,-2.8276729137

H,0,-0.9586442663,0.5864504583,-2.8265479373

C,0,0.2915808919,0.9452857949,2.1749439625

H,0,-0.5908547506,0.9659046727,2.8232643661

H,0,0.2966492192,1.8541356809,1.5714260697 
H,0,1.1709162171,0.9594969907,2.8276235437

\section{propane}

B3LYP ENERGY = -119.1810826

Zero-point correction=

0.102894

Thermal correction to Energy= 0.107444

Thermal correction to Enthalpy= 0.108388

Thermal correction to Gibbs Free Energy= $\quad 0.077860$

Sum of electronic and zero-point Energies= $-119.078189$

Sum of electronic and thermal Energies= $-119.073639$

Sum of electronic and thermal Enthalpies= $\quad-119.072695$

Sum of electronic and thermal Free Energies= $-119.103222$

Charge $=0$ Multiplicity $=1$

H,0,0.504325597,0.8000907605,-1.9910574937

C,0,0.5039301978,0.7994634768,-0.8974245145

H,0,1.5468781177,0.7985924572,-0.5641760149

H,0,0.0533120819,1.7400401276,-0.5641760149

C,0,-0.2557030928,-0.4056619042,-0.3357220002

H,0,0.1970116301,-1.3294850886,-0.7138625217

H,0,-1.284440244,-0.3956734042,-0.7138625217

C,0,-0.2772472523,-0.4398407821,1.1950448953

H,0,0.7378431441,-0.4849065587,1.6029128247

H,0,-0.8250865504,-1.3089641485,1.5699186342

H,0,-0.7557228918,0.4565411118,1.6029128247

\section{ethane}

B3LYP ENERGY = -79.8565419

Zero-point correction= 0.074310

Thermal correction to Energy= 0.077797

Thermal correction to Enthalpy= 0.078741

Thermal correction to Gibbs Free Energy= 0.051847 
Sum of electronic and zero-point Energies=

Sum of electronic and thermal Energies=

Sum of electronic and thermal Enthalpies=

Sum of electronic and thermal Free Energies=

Charge $=0$ Multiplicity $=1$

$\mathrm{H}, 0,0.3807317559,0.6035694582,-1.3721570811$

C,0,0.3802835607,0.6028589397,-0.2784559604

H,0,1.4232479423,0.603154089,0.0509228203

H,0,-0.068663547,1.5442522066,0.0509228203

C,0,-0.3802835607,-0.6028589397,0.2784559604

H,0,0.068663547,-1.5442522066,-0.0509228203

H,0,-1.4232479423,-0.603154089,-0.0509228203

H,0,-0.3807317559,-0.6035694582,1.3721570811
$-79.782232$

$-79.778745$

$-79.777801$

$-79.804695$

\section{trans-2-pentene}

B3LYP ENERGY = -196.5986713

Zero-point correction= 0.135909

Thermal correction to Energy= 0.142650

Thermal correction to Enthalpy= 0.143594

Thermal correction to Gibbs Free Energy=

0.106077

Sum of electronic and zero-point Energies= $-196.462762$

Sum of electronic and thermal Energies= $-196.456021$

Sum of electronic and thermal Enthalpies= $-196.455077$

Sum of electronic and thermal Free Energies= $-196.492594$

Charge $=0$ Multiplicity $=1$

C,0,-1.1611615423,0.2828767439,0.3393057628

H,0,-1.1572839331,0.2825430631,1.4293773273

C,0,0.0080460492,0.279992368,-0.3012359336

H,0,0.0070838389,0.2776148586,-1.3923201522

C,0,-2.5117337968,0.2911196725,-0.3158783488

H,0,-3.0906824224,1.1730972551,-0.0193134495 
H,0,-3.1009508642,-0.5843940657,-0.0204652124

H,0,-2.4287084153,0.2915708272,-1.4054929306

C,0,1.3616159767,0.2655456763,0.353229679

H,0,1.9133783839,1.1689261021,0.0619797374

H,0,1.2425503331,0.3118920028,1.4407850266

C,0,2.1992993191,-0.9660212866,-0.0277260132

H,0,2.342536135,-1.0259212302,-1.1110571306

H,0,1.7070689845,-1.8880363377,0.2933344487

H,0,3.1886119243,-0.92837152,0.4370014583

\section{trans-2,3,4-hexatriene}

B3LYP ENERGY $=-233.4370224$

Zero-point correction=

0.116643

Thermal correction to Energy=

0.124232

Thermal correction to Enthalpy= 0.125176

Thermal correction to Gibbs Free Energy=

0.085280

Sum of electronic and zero-point Energies= $-233.320379$

Sum of electronic and thermal Energies= $-233.312791$

Sum of electronic and thermal Enthalpies= $-233.311847$

Sum of electronic and thermal Free Energies= $-233.351743$

C,0,0.0002497435,0.0002391646,-0.6328920906

C,0,-0.0002401296,-0.0006694917,0.6328932156

C,0,0.0044385131,-0.0015014834,1.9512997851

H,0,-0.952650171,-0.0262401114,2.4708358832

C,0,-0.004428113,0.0010597826,-1.9512986204

C,0,1.2390716401,0.0303604234,2.8102660679

H,0,1.2797175549,-0.8493121852,3.4623287576

H,0,1.2337233852,0.910025249,3.4635132183

H,0,2.1456187657,0.0543701913,2.2045666722

H,0,0.9526696936,0.0254605935,-2.4708338535

C,0,-1.2390852327,-0.0297758435,-2.8102676351 
H,0,-2.1456099854,-0.054723159,-2.2045726258

H,0,-1.2336460513,-0.9085923658,-3.4646600009

H,0,-1.2798617205,0.8507364755,-3.4611823852

\author{
1,3-butadiene \\ B3LYP ENERGY = -156.0408103 \\ Zero-point correction= \\ 0.084757 \\ Thermal correction to Energy= \\ 0.089413 \\ Thermal correction to Enthalpy= \\ 0.090357 \\ Thermal correction to Gibbs Free Energy= \\ 0.058958 \\ Sum of electronic and zero-point Energies= \\ $-155.956054$ \\ Sum of electronic and thermal Energies= \\ $-155.951397$ \\ Sum of electronic and thermal Enthalpies= \\ $-155.950453$ \\ Sum of electronic and thermal Free Energies= \\ $-155.981852$ \\ C,0,0.6531967159,0.,0.3213901025 \\ H,0,0.6560591677,0.,1.4095959704 \\ C,0,1.817529979,0.,-0.3378480692 \\ H,0,2.767154468,0.,0.1834372111 \\ H,0,1.8540510052,0.,-1.4228389833 \\ C,0,-0.6531967159,0.,-0.3213901025 \\ H,0,-0.6560591677,0.,-1.4095959704 \\ C,0,-1.817529979,0.,0.3378480692 \\ H,0,-2.767154468,0.,-0.1834372111 \\ H,0,-1.8540510052,0.,1.4228389833
}

\title{
1,2,3-butatriene
}

B3LYP ENERGY = -154.777635

Zero-point correction=

0.059864

Thermal correction to Energy=

0.064585

Thermal correction to Enthalpy=

0.065529

Thermal correction to Gibbs Free Energy $=\quad 0.035249$ 
Sum of electronic and zero-point Energies=

Sum of electronic and thermal Energies=

Sum of electronic and thermal Enthalpies=

Sum of electronic and thermal Free Energies=

C,0,1.6582853612,0.,1.0214465367

H,0,1.6545924676,0.,2.1072135871

H,0,2.6263042404,0.,0.5296710038

C,0,0.5387987656,0.,0.3318814397

C,0,-0.5387987656,0.,-0.3318814397

C,0,-1.6582853612,0.,-1.0214465367

H,0,-2.6263042404,0.,-0.5296710038

H,0,-1.6545924676,0.,-2.1072135871
$-154.717771$

$-154.713050$

$-154.712106$

$-154.742386$

\section{n-pentane}

B3LYP ENERGY = -197.8298083

Zero-point correction=

0.159731

Thermal correction to Energy=

0.166844

Thermal correction to Enthalpy=

0.167788

Thermal correction to Gibbs Free Energy=

0.130031

Sum of electronic and zero-point Energies= $-197.670077$

Sum of electronic and thermal Energies= $-197.662964$

Sum of electronic and thermal Enthalpies= $-197.662020$

Sum of electronic and thermal Free Energies= $-197.699777$

H,0,0.4155703836,2.5255984333,-1.4001962868

C,0,0.4162353801,2.5281328592,-0.3055152781

H,0,1.4603792469,2.5255984333,0.0232559771

H,0,-0.0328884498,3.4692248387,0.0241400044

C,0,-0.3419428348,1.3195662623,0.2509848161

H,0,-1.3908942056,1.3689083629,-0.0660452901

H,0,-0.353920159,1.3689083629,1.3467327844

C,0,0.2531325056,-0.0237806574,-0.1857983525 
H,0,1.3033856386,-0.0737076121,0.1309473509

H,0,0.2657713652,-0.0737076121,-1.2827029715

C,0,-0.498250967,-1.2406338435,0.3657144252

H,0,-1.5471425274,-1.1903120966,0.0486404184

H,0,-0.5101684808,-1.1903120966,1.4614184929

C,0,0.1045476888,-2.5770580799,-0.0767376292

H,0,1.1430483494,-2.6720236345,0.2561757192

H,0,0.0982394861,-2.6720236344,-1.1672765447

H,0,-0.4537112833,-3.4235109881,0.3330224568

\section{n-hexane}

B3LYP ENERGY = -237.1541295

Zero-point correction $=$

0.188031

Thermal correction to Energy=

0.196496

Thermal correction to Enthalpy=

0.197441

Thermal correction to Gibbs Free Energy=

0.155975

Sum of electronic and zero-point Energies= $-236.966098$

Sum of electronic and thermal Energies= $-236.957633$

Sum of electronic and thermal Enthalpies= $-236.956689$

Sum of electronic and thermal Free Energies= $-236.998155$

H,0,0.5215850101,3.164861518,-1.4782396009

C,0,0.5222636063,3.1639724648,-0.3835080263

H,0,1.5665893313,3.164861518,-0.0551462423

H,0,0.0691204377,4.1020413745,-0.0507564422

C,0,-0.2299201439,1.9497597578,0.1688347027

H,0,-1.2791044455,1.994991649,-0.147861576

H,0,-0.2418265342,1.994991649,1.2647099098

C,0,0.3723454474,0.6111027815,-0.2734202919

H,0,1.4228024821,0.5669189779,0.0430784566

H,0,0.3848213095,0.566918978,-1.3704507346

C,0,-0.3723454474,-0.6111027815,0.2734202919 
H,0,-1.4228024821,-0.5669189779,-0.0430784566

H,0,-0.3848213095,-0.566918978,1.3704507346

C,0,0.2299201439,-1.9497597578,-0.1688347027

H,0,1.2791044455,-1.994991649,0.147861576

H,0,0.2418265342,-1.994991649,-1.2647099098

C,0,-0.5222636063,-3.1639724648,0.3835080263

H,0,-0.0691204377,-4.1020413745,0.0507564422

H,0,-1.5665893313,-3.164861518,0.0551462423

H,0,-0.5215850101,-3.164861518,1.4782396009

\section{cis-2,3,4-hexatriene}

B3LYP ENERGY $=-233.4369871$

Zero-point correction= 0.116637

Thermal correction to Energy= 0.124205

Thermal correction to Enthalpy= 0.125149

Thermal correction to Gibbs Free Energy= 0.085192

Sum of electronic and zero-point Energies= $-233.320350$

Sum of electronic and thermal Energies= $-233.312782$

Sum of electronic and thermal Enthalpies= $-233.311838$

Sum of electronic and thermal Free Energies= $-233.351796$

C,0,-1.4737698622,-0.0006325972,1.373823667

H,0,-1.4660919128,0.0003162416,2.4631638048

C,0,-0.316951576,0.0000694603,0.7417758365

C,0,0.794948724,0.0012887354,0.1369598904

C,0,1.9541030386,0.0027083835,-0.4907900722

H,0,2.8644525035,0.0040725761,0.1075286327

C,0,2.1278533565,0.0008667503,-1.9848972114

H,0,1.1658540716,-0.000773058,-2.4988140991

H,0,2.6959514046,-0.8789585876,-2.3076548418

H,0,2.6942365223,0.8809224824,-2.3099944991

C,0,-2.8224951604,-0.0032576416,0.7078851876 
H,0,-3.4040595722,0.8759361535,1.0075237779

Н,0,-3.4012233367,-0.8839430327,1.0086612317

H,0,-2.731250803,-0.0038313196,-0.3789577949

1,2,3-Cyclobutatriene (one imaginary frequency)

B3LYP ENERGY = -153.3081503

Zero-point correction=

0.035608

Thermal correction to Energy= 0.038843

Thermal correction to Enthalpy= 0.039788

Thermal correction to Gibbs Free Energy=

0.011363

Sum of electronic and zero-point Energies=

$-153.272542$

Sum of electronic and thermal Energies=

$-153.269307$

Sum of electronic and thermal Enthalpies=

$-153.268363$

Sum of electronic and thermal Free Energies=

$-153.296787$

C, $0.000000,0.795588,-0.854165$

C, $0.000000,-0.795588,-0.854165$

C, $0.000000,0.689220,0.618018$

C,0.000000,-0.689220,0.618018

$\mathrm{H}, 0.000000,1.439843,1.416884$

H,0.000000,-1.439843,1.416884

\section{1,2,3-Cyclopentatriene}

B3LYP ENERGY = -192.7655845

Zero-point correction=

0.066015

Thermal correction to Energy= 0.070679

Thermal correction to Enthalpy= 0.071623

Thermal correction to Gibbs Free Energy $=\quad 0.039082$

Sum of electronic and zero-point Energies= $-192.699569$

Sum of electronic and thermal Energies= $-192.694906$

Sum of electronic and thermal Enthalpies= $-192.693962$

Sum of electronic and thermal Free Energies= $-192.726503$ 
C,-0.644421,-1.072828,0.000000

C,0.644242,-1.072454,0.000000

C,-1.213025,0.146616,0.000000

C,1.213180,0.146740,0.000000

C,0.000000,1.108199,0.000000

$\mathrm{H},-2.247802,0.457504,0.000000$

H,2.247954,0.457577,0.000000

$\mathrm{H},-0.000003,1.773639,0.875993$

H,-0.000003,1.773639,-0.875993

\section{1,2,3-Cyclohexatriene}

B3LYP ENERGY = -232.1438871

Zero-point correction=

0.096210

Thermal correction to Energy=

0.101690

Thermal correction to Enthalpy=

0.102634

Thermal correction to Gibbs Free Energy=

0.067708

Sum of electronic and zero-point Energies= $-232.047677$

Sum of electronic and thermal Energies= $-232.042198$

Sum of electronic and thermal Enthalpies= $-232.041253$

Sum of electronic and thermal Free Energies= $-232.076179$

C,0.638174,-1.276961,-0.053393

C,1.504040,-0.280517,-0.078454

C,-0.638135,-1.276979,0.053409

C,-1.504044,-0.280562,0.078352

C,-0.764066,1.075398,-0.148241

C,0.764038,1.075403,0.148251

H,2.585697,-0.309944,-0.123234

$\mathrm{H},-2.585681,-0.309983,0.123608$

H,-0.938232,1.353379,-1.196865

H,-1.239933,1.849249,0.460888

H,1.239877,1.849304,-0.460833 
H,0.938221,1.353308,1.196895

\section{1,2,3-Cycloheptatriene}

B3LYP ENERGY = -271.5005972

Zero-point correction=

0.125677

Thermal correction to Energy=

0.131969

Thermal correction to Enthalpy=

0.132914

Thermal correction to Gibbs Free Energy=

0.096036

Sum of electronic and zero-point Energies=

$-271.374920$

Sum of electronic and thermal Energies=

$-271.368628$

Sum of electronic and thermal Enthalpies= $\quad-271.367684$

Sum of electronic and thermal Free Energies $=\quad-271.404561$

C,-0.634627,-1.399566,-0.093104

C,0.634271,-1.399558,-0.092729

C,1.706142,-0.639449,0.041974

C,-1.706265,-0.639143,0.041940

C,1.365188,0.840553,0.213930

C,0.000114,1.302174,-0.384900

C,-1.364954,0.840740,0.214083

H,2.738394,-0.971774,0.053905

H,-2.738612,-0.971187,0.053877

H,1.413404,1.092954,1.281993

H,2.146999,1.441459,-0.266849

H,0.000202,2.395635,-0.336962

H,0.000034,1.043550,-1.448927

H,-1.412907,1.092938,1.282212

H,-2.146725,1.441919,-0.266414

\section{1,2,3-Cyclooctatriene}

B3LYP ENERGY = -310.8292494

Zero-point correction=

0.154564 
$\begin{array}{lc}\text { Thermal correction to Energy= } & 0.162222 \\ \text { Thermal correction to Enthalpy= } & 0.163167 \\ \text { Thermal correction to Gibbs Free Energy= } & 0.122777 \\ \text { Sum of electronic and zero-point Energies= } & -310.674685 \\ \text { Sum of electronic and thermal Energies= } & -310.667027 \\ \text { Sum of electronic and thermal Enthalpies= } & -310.666083 \\ \text { Sum of electronic and thermal Free Energies= } & -310.706472 \\ \text { C,-0.821701,-1.488146,-0.089184 } & \\ \text { C,0.434637,-1.558793,-0.228550 } & \\ \text { C,1.669230,-1.108950,-0.145448 } & \\ \text { C,-1.918715,-0.795258,0.143138 } & \\ \text { C,-1.689930,0.704386,0.360221 } \\ \text { C,1.822204,0.296434,0.440080 } \\ \text { C,-0.565268,1.291924,-0.539974 } \\ \text { C,0.908697,1.455522,-0.062393 } \\ \text { H,2.555957,-1.706051,-0.332408 } \\ \text { H,-2.925442,-1.198926,0.153064 } \\ \text { H,-2.623983,1.227930,0.130704 } \\ \text { H,-1.493213,0.900585,1.422348 } \\ \text { H,1.689367,0.184596,1.524096 } \\ \text { H,2.857479,0.626666,0.307928 } \\ \text { H,-0.571481,0.737042,-1.481774 } \\ \text { H,-0.868866,2.311966,-0.802374 } \\ \begin{array}{l}\text { H,0.927973,2.215793,0.727439 } \\ \text { H,1.417290,1.917688,-0.916360 }\end{array}\end{array}$

\section{1,2,3-Cyclononatriene}

B3LYP ENERGY = -350.1778871

Zero-point correction= 0.183535

Thermal correction to Energy= 0.192111

Thermal correction to Enthalpy= 0.193055 
Thermal correction to Gibbs Free Energy=

Sum of electronic and zero-point Energies=

Sum of electronic and thermal Energies=

Sum of electronic and thermal Enthalpies=

Sum of electronic and thermal Free Energies=

C,1.227541,0.023383,1.907009

H,1.995083,0.091000,2.674306

C,1.535459,-0.102652,0.632232

C,1.535459,-0.102652,-0.632232

C,-0.221780,0.127616,2.347646

$\mathrm{H},-0.419144,1.168153,2.645449$

H,-0.348874,-0.463583,3.262932

C,1.227541,0.023383,-1.907009

H,1.995083,0.091000,-2.674306

C,-1.262971,-0.334958,1.310872

$\mathrm{H},-2.235459,-0.359372,1.812630$

C, $-0.221780,0.127616,-2.347646$

H, $-0.348874,-0.463583,-3.262932$

$\mathrm{H},-0.419144,1.168153,-2.645449$

H,-1.033989,-1.374601,1.055490

C,-1.379900,0.500957,0.000000

$\mathrm{H},-2.345235,1.016744,0.000000$

$\mathrm{H},-0.629582,1.293662,0.000000$

C,-1.262971,-0.334958,-1.310872

H,-1.033989,-1.374601,-1.055490

H,-2.235459,-0.359372,-1.812630
0.150625

$-349.994352$

$-349.985776$

$-349.984832$

$-350.027262$ 


$$
\begin{aligned}
& \text { Thermal correction to Energy }=\quad 0.089851 \\
& \text { Thermal correction to Enthalpy= } \quad 0.090795 \\
& \text { Thermal correction to Gibbs Free Energy }=\quad 0.061057 \\
& \text { Sum of electronic and zero-point Energies }=\quad-155.931261 \\
& \text { Sum of electronic and thermal Energies= } \quad-155.927430 \\
& \text { Sum of electronic and thermal Enthalpies= } \quad-155.926486 \\
& \text { Sum of electronic and thermal Free Energies }=\quad-155.956224 \\
& \text { Charge }=0 \text { Multiplicity }=1 \\
& \text { H,0,-0.539422194,0.6607458254,-1.709598633 } \\
& \text { C,0,-0.5428748258,0.6594301002,-0.6152959512 } \\
& \text { C,0,0.8213006621,0.6586391056,0.0517447925 } \\
& \text { C,0,-0.7312760709,-0.7559488068,0.0438970472 } \\
& \text { C,0,0.6609030819,-0.5463601058,0.6129563799 } \\
& \text { H,0,1.2878280349,-1.1633120964,1.2467778322 } \\
& \text { H,0,-1.2434279588,1.4254327501,-0.2689186147 } \\
& \text { H,0,-1.5421080683,-0.8184246444,0.776126692 } \\
& \text { H,0,-0.8381023036,-1.5831115691,-0.6645533263 } \\
& \text { H,0,1.6269154065,1.3841079752,0.0603524397 }
\end{aligned}
$$

\section{cyclopentene}

B3LYP ENERGY = -195.3819435

Zero-point correction= 0.116189

Thermal correction to Energy= 0.120848

Thermal correction to Enthalpy= 0.121792

Thermal correction to Gibbs Free Energy= 0.088798

Sum of electronic and zero-point Energies= $-195.265755$

Sum of electronic and thermal Energies= $-195.261096$

Sum of electronic and thermal Enthalpies= $-195.260152$

Sum of electronic and thermal Free Energies= $-195.293146$

Charge $=0$ Multiplicity $=1$

H,0,0.075787924,0.0304093244,-2.3476286632 
C,0,0.0736426557,0.0268320153,-1.2633751462

C,0,1.1639453636,0.0268320162,-0.4967606982

C,0,0.8241053818,0.0784827674,0.9749007344

C,0,-0.6997908976,-0.2135107329,0.9952641941

C,0,-1.1962368934,0.0784827658,-0.4456438235

H,0,2.1835246052,0.030409326,-0.8656352858

H,0,1.3954473504,-0.6414320911,1.5694422383

H,0,1.0554599142,1.0703374442,1.3863648698

H,0,-1.2311522665,0.3685586673,1.7509827177

H,0,-0.8623238149,-1.2699010097,1.2264235223

H,0,-1.661711718,1.0703374421,-0.524134862

H,0,-1.9490256557,-0.6414320938,-0.7821261004

\section{cyclohexene}

B3LYP ENERGY = -234.7132201

Zero-point correction=

0.145561

Thermal correction to Energy=

0.151090

Thermal correction to Enthalpy=

0.152034

Thermal correction to Gibbs Free Energy=

0.117560

Sum of electronic and zero-point Energies= $-234.567659$

Sum of electronic and thermal Energies= $-234.562130$

Sum of electronic and thermal Enthalpies= $-234.561186$

Sum of electronic and thermal Free Energies= $-234.595660$

Charge $=0$ Multiplicity $=1$

C,0,-0.6822113446,0.1331252014,-1.2907979275

C,0,-0.6842366682,0.1292030955,1.2350601243

C,0,1.4951425804,0.1298043868,-0.049473431

C,0,0.7165268731,-0.48581293,1.1228632178

C,0,0.6468658725,0.257226702,-1.2902297835

C,0,-1.4964444672,-0.1154264774,-0.0455940764

H,0,-0.5885557665,1.2094356081,1.3991622066 
H,0,1.8786582918,1.1210953627,0.2313237564

H,0,0.620900562,-1.5666483358,0.9627474979

H,0,-1.8763158697,-1.1469648412,-0.0602733777

H,0,-1.2153098034,-0.2751021152,2.1021941238

H,0,2.3826550605,-0.4738657651,-0.2720438123

H,0,1.2722566027,-0.3538127131,2.056224435

H,0,-2.387145894,0.5234620866,-0.0558897241

H,0,1.1638476367,0.4589410117,-2.2250963644

H,0,-1.2248478961,0.2147398313,-2.2293174831

\section{cycloheptene}

B3LYP ENERGY = -274.0287567

Zero-point correction= 0.174324

Thermal correction to Energy= 0.181048

Thermal correction to Enthalpy= 0.181992

Thermal correction to Gibbs Free Energy= 0.144637

Sum of electronic and zero-point Energies= $-273.854433$

Sum of electronic and thermal Energies= $-273.847709$

Sum of electronic and thermal Enthalpies= $-273.846765$

Sum of electronic and thermal Free Energies= $-273.884120$

Charge $=0$ Multiplicity $=1$

C,0,-0.984211032,0.1429993059,-1.3614835914

C,0,-0.9824156288,0.1401650786,1.2064389634

C,0,1.4953629761,0.1480537205,0.4269090713

C,0,0.499797419,0.2808300164,1.5914754926

C,0,1.182498133,-0.974699826,-0.5767114889

C,0,-1.3922843701,0.8568235221,-0.0912984735

H,0,-1.5933776729,0.5170135619,2.0338312042

H,0,1.5439280725,1.0917952251,-0.1270439434

H,0,0.7295258369,-0.4728263321,2.3538747104

H,0,-2.4827457116,0.9601995468,-0.1056537968 
H,0,-1.6542516469,0.2866985349,-2.2066307557

H,0,-1.2378889459,-0.9198356078,1.1015463143

H,0,2.4966138981,-0.0094962473,0.842290724

H,0,0.6600995244,1.2536397139,2.0710275047

H,0,-1.0071871035,1.8868221991,-0.0936174861

H,0,0.9643929961,-1.9108676812,-0.0426492705

C,0,0.0694832209,-0.6569736011,-1.551230317

H,0,0.1667750195,-1.1224933384,-2.5298534842

H,0,2.0847314252,-1.183838874,-1.1617196598

\section{(Z)-cyclooctene}

B3LYP ENERGY = -313.3475592

Zero-point correction= 0.203359

Thermal correction to Energy= 0.211105

Thermal correction to Enthalpy= 0.212049

Thermal correction to Gibbs Free Energy= 0.171661

Sum of electronic and zero-point Energies= $-313.144201$

Sum of electronic and thermal Energies= $-313.136454$

Sum of electronic and thermal Enthalpies= $-313.135510$

Sum of electronic and thermal Free Energies= $-313.175898$

Charge $=0$ Multiplicity $=1$

C,0,1.5214914969,-0.0405544358,0.9920037195

H,0,1.5184355775,-0.040838342,2.0892598313

H,0,2.578225147,-0.0415142229,0.6974067056

C,0,0.9104569273,1.2744571633,0.4791672251

H,0,0.9903917486,1.3071581479,-0.6119637332

H,0,1.5356374995,2.0970723774,0.8457491439

C,0,-1.4888723091,0.101921837,-1.0075573019

H,0,-2.2396240553,0.5262317253,-1.6710167476

C,0,-0.5813999759,-0.696411853,-1.5810942683

H,0,-0.6924465743,-0.8531082323,-2.6537360602 
C,0,0.5962824169,-1.4486710553,-1.0047195974

H,0,0.4561083781,-2.5063950593,-1.266779542

H,0,1.4934091576,-1.1462091797,-1.565200813

C,0,0.8929717823,-1.3628689343,0.5011592534

H,0,1.5983557912,-2.1660303726,0.7429154297

H,0,-0.0109947239,-1.5988010788,1.0654565549

C,0,-0.5400211158,1.5747624646,0.8801443377

H,0,-0.8124071669,2.5465814161,0.4520552964

H,0,-0.6067231393,1.6915102933,1.9689221832

C,0,-1.5924641508,0.5262658378,0.4407773795

H,0,-1.5331137578,-0.3419246311,1.0984170453

H,0,-2.5859243115,0.9528610134,0.6092302208

\section{(Z)-cyclononene}

B3LYP ENERGY = -352.691959

Zero-point correction= 0.231776

Thermal correction to Energy= 0.240729

Thermal correction to Enthalpy= $\quad 0.241673$

Thermal correction to Gibbs Free Energy=

0.198613

Sum of electronic and zero-point Energies= $-352.437419$

Sum of electronic and thermal Energies= $-352.428467$

Sum of electronic and thermal Enthalpies= $-352.427523$

Sum of electronic and thermal Free Energies= $-352.470582$

Charge $=0$ Multiplicity $=1$

C,0,1.6925733115,0.0284540114,-0.6576251224

H,0,1.6993247204,0.0164431488,0.436983796

H,0,2.7465482775,0.0281813508,-0.9594330582

C,0,1.079109456,-1.2776356278,-1.1883127869

H,0,1.7185371835,-2.1010175763,-0.8451692523

H,0,1.1569794789,-1.2806928536,-2.2834447179

C,0,-0.3752118563,-1.6292370324,-0.8211971991 
H,0,-0.5748908177,-2.608445317,-1.2702578556

H,0,-1.0668913613,-0.9446178825,-1.321674125

C,0,-0.7333485264,-1.7110104428,0.6849639576

$\mathrm{H}, 0,0.1733985956,-1.8524751743,1.2837581989$

H,0,-1.3337864447,-2.6133773375,0.8379972115

C,0,-1.5559527439,-0.5235682682,1.2581744451

H,0,-2.1358461769,-0.89720533,2.1076502765

H,0,-2.2931169736,-0.2224997711,0.5049339771

C,0,-0.7745329041,0.6793957132,1.7363098501

$\mathrm{H}, 0,-0.6168702805,0.7373198159,2.8114663804$

C,0,-0.2674695304,1.6628369154,0.9875636266

H,0,0.2752534232,2.459327561,1.494732003

C,0,-0.2902766024,1.7387192465,-0.5124632386

H,0,-0.5279043769,2.7616800404,-0.8268435273

H,0,-1.0811585169,1.1019442656,-0.9134365519

C,0,1.0755665465,1.3522918346,-1.1394445409

$\mathrm{H}, 0,0.970063692,1.3390855393,-2.2312684008$

H,0,1.7876166742,2.1548714214,-0.9138083387

1,2-Cyclopentadiene; transition state for racemization (structure 23)

Basis set 6-311+G(d,p)

Optimized structure

\begin{tabular}{|c|c|c|c|c|}
\hline \multirow{2}{*}{$\begin{array}{l}\text { Center } \\
\text { Number }\end{array}$} & \multirow{2}{*}{$\begin{array}{l}\text { Atomic } \\
\text { Number }\end{array}$} & \multirow{2}{*}{$\begin{array}{l}\text { Atomic } \\
\text { Type }\end{array}$} & \multicolumn{2}{|c|}{ Coordinates (Angstroms) } \\
\hline & & & $\mathrm{X}$ & $\mathrm{Z}$ \\
\hline 1 & 6 & 0.000000 & 0.000000 & 0.000000 \\
\hline 2 & 6 & 0.000000 & 0.000000 & 2.331894 \\
\hline 3 & 6 & 0.723658 & 0.000000 & 1.165947 \\
\hline 4 & 1 & 0.372534 & 0.000000 & -1.014325 \\
\hline 5 & 1 & 0.372534 & 0.000000 & 3.346219 \\
\hline 6 & 6 & -1.472009 & 0.000000 & 0.382899 \\
\hline 7 & 1 & -1.988220 & -0.877809 & -0.022611 \\
\hline 8 & 1 & -1.988220 & 0.877809 & -0.022611 \\
\hline 9 & 6 & -1.472009 & 0.000000 & 1.948995 \\
\hline
\end{tabular}




$\begin{array}{llllll}10 & 1 & 0 & -1.988220 & -0.877809 & 2.354505 \\ 11 & 1 & 0 & -1.988220 & 0.877809 & 2.354505\end{array}$

$\mathrm{E}(\mathrm{UB}+\mathrm{HF}-\mathrm{LYP})=-194.049622465 \mathrm{~S} * * 2=1.0420$

Imaginary mode $-156.9664 \mathrm{~cm}^{-1}$

Zero-point correction $=0.088359$ (Hartree/Particle) Thermal correction to Energy= 0.092651

Thermal correction to Enthalpy= 0.093595

Thermal correction to Gibbs Free Energy $=0.062251$

Sum of electronic and zero-point Energies= $\quad-193.961264$

Sum of electronic and thermal Energies $=\quad-193.956972$

Sum of electronic and thermal Enthalpies= $\quad-193.956028$

Sum of electronic and thermal Free Energies $=\quad-193.987372$

Bicyclo[3.2.1]octa-2,3-diene; transition state for racemization (structure 24)

Basis set 6-311+G(d,p)

Optimized structure

\begin{tabular}{cccccc} 
Center & \multicolumn{2}{c}{ Atomic } & \multicolumn{2}{c}{ Atomic } & \multicolumn{3}{c}{ Coordinates } \\
Number & Number & Type & X & Y & Z \\
\hline------- \\
1 & 1 & 0 & 0.000000 & 0.000000 & 0.000000 \\
2 & 6 & 0 & 0.000000 & 0.000000 & 1.091771 \\
3 & 6 & 0 & 1.419940 & 0.000000 & 1.700749 \\
4 & 6 & 0 & 1.112174 & 0.277287 & 3.206492 \\
5 & 6 & 0 & -0.195103 & 1.129493 & 3.206492 \\
6 & 6 & 0 & -0.573007 & 1.299189 & 1.700749 \\
7 & 1 & 0 & -0.566549 & -0.869081 & 1.442559 \\
8 & 1 & 0 & 1.954113 & -0.942212 & 1.558718 \\
9 & 1 & 0 & 0.952150 & -0.671595 & 3.727321 \\
10 & 1 & 0 & 1.941561 & 0.784360 & 3.702010 \\
11 & 1 & 0 & -0.065845 & 2.092975 & 3.702010 \\
12 & 1 & 0 & -0.998716 & 0.600163 & 3.727321 \\
13 & 1 & 0 & -1.650655 & 1.407714 & 1.558718 \\
14 & 6 & 0 & 2.212560 & 1.155555 & 1.092333 \\
15 & 1 & 0 & 3.277497 & 1.061043 & 0.913385 \\
16 & 6 & 0 & 1.495961 & 2.294794 & 0.853639 \\
17 & 6 & 0 & 0.164425 & 2.490721 & 1.092333 \\
18 & 1 & 0 & -0.351798 & 3.426957 & 0.913385 \\
------------------------
\end{tabular}


$\mathrm{E}(\mathrm{UB}+\mathrm{HF}-\mathrm{LYP})=-310.817049994 \quad \mathrm{~S} * * 2=1.0419$

One imaginary mode $-346.8251 \mathrm{~cm}^{-1}$

Zero-point correction=

Thermal correction to Energy=

Thermal correction to Enthalpy=

Thermal correction to Gibbs Free Energy=

Sum of electronic and zero-point Energies=

Sum of electronic and thermal Energies=

Sum of electronic and thermal Enthalpies=

Sum of electronic and thermal Free Energies=
0.153961 (Hartree/Particle)

0.159930

0.160874
0.124350

$-310.663089$

$-310.657120$

$-310.656176$

$-310.692700$ 


\section{Butatriene Bond Strength Calculation B3LYP/6-311+G(d,p)}

butadienyl radical $\quad-155.358444$

1,3-butadiene S-trans $\quad-156.040790$

1,2,3-butatriene $\quad-154.777541$

\section{Allene Bending Potential B3L YP/6-311+G(d,p)}

Energies in $\mathrm{kcal} / \mathrm{mol}$

$\begin{array}{lc}\text { E }_{\text {relative }} & \text { Angle } \\ --------------- \\ 0.000 & 180.00 \\ 0.923 & 168.33 \\ 4.510 & 156.66 \\ 10.80 & 144.99 \\ 19.95 & 133.33 \\ 32.41 & 121.66 \\ 48.49 & 110.00\end{array}$

Butatriene Bending Potential B3LYP/6-311+G(d,p)

Energies in $\mathrm{kcal} / \mathrm{mol}$

\begin{tabular}{lc}
\multicolumn{1}{c}{ Erelative } & Angle \\
------------- \\
0.0000 & 180.00 \\
2.503 & 168.33 \\
10.80 & 156.66 \\
24.49 & 145.00 \\
42.95 & 133.33 \\
70.51 & 121.66 \\
132.2 & 110.00
\end{tabular}

